\title{
Pseudoterranovosis y sushi
}

\author{
Leonor Jofré M., Patricia Neira O., Isabel Noemí H. y José L. Cerva C.
}

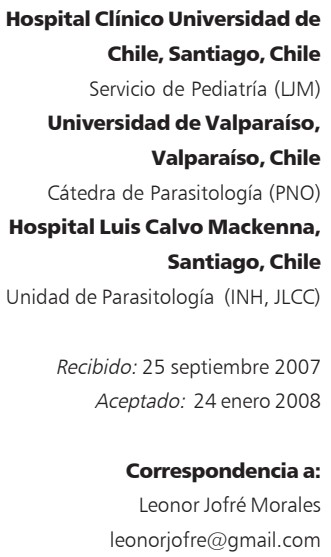

Correspondencia a: Leonor Jofré Morales leonorjofre@gmail.com

\section{Pseudoterranovosis and sushi}

A case of accidental ingestion of Pseudoterranova decipiens is reported, on account of this patient we review main concepts related to this emerging pathology in Chile. Consumption of raw fish in different preparations such as sushi or sashimi, cebiche and seafood is becoming more and more popular. Through intermediary hosts used in the preparation, one can acquire the third stage larval of Anisakis simplex or $P$. decipiens. These parasites frequently cause gastritis, acutely after the consumption of raw fish, or more rarely intestinal involvement. It may also cause allergic symptoms such as urticaria, angioedema and anaphylactic shock. The larvae is eliminated spontaneously in most cases; others need to be removed by upper gastrointestinal endoscopic examination. In Chile, the most common species is $P$. decipiens, which are generally no invasive, rarely migrate beyond the stomach and cause mild or no symptoms. Prevention strategies include avoid eating raw fish or undercooked seafood containing the parasite, to cook food with high temperatures or to freeze raw fish prior to preparation of meals, which inactivates the larvae.

Key words: Anisakidosis, anisakiosis, pseudoterranovosis, Anisakis simplex, Pseudoterranova decipiens, foodborne parasitic infections, fishborne parasitic infections, zoonoses.

Palabras clave: anisakidosis, anisakiosis, pseudoterranovosis, Anisakis simplex, Pseudoterranova decipiens, enfermedades parasitarias transmitidas por alimentos, enfermedades parasitarias transmitidas por peces y mariscos, zoonosis.

\section{Introducción}

e estima que las infecciones parasitarias transmitidas por alimentos afectan a más de 50 millones de personas en el mundo, cifra probablemente sub-estimada por la dificultad en el diagnóstico y una sub-notificación. La mayor incidencia de estas infecciones se encuentra en el Este y Sudeste asiático, situación que se está haciendo cada vez más frecuente en nuestro medio con los viajes a destinos exóticos y la incorporación de la comida oriental a los menús tradicionales. Las infecciones parasitarias son el resultado de la ingesta de alimentos crudos o mal cocidos. Comentario editorial en página 196. La mayoría de estos cuadros son producidos por nemátodos, tremátodos, céstodos y protozoos ${ }^{1}$. Presentamos el caso de una paciente que tras visitar un restaurante de comida japonesa, elimina con la tos una forma juvenil de nemátodo.

\section{Caso Clínico}

Paciente de 30 años, sexo femenino, sana, residente en la ciudad de Santiago. Asistió a un restaurante de comida japonesa, donde degustó sushi de variados sabores, algunos de ellos preparados con salmón crudo. Al día siguiente, comenzó con tos y estornudos, se limpió la boca con un pañuelo y se percató de la presencia de un elemento blanquecino móvil de pequeño tamaño. Lo llevó a un centro asistencial, desde donde se derivó la muestra al laboratorio de referencia de Parasitología, para su identificación. Se informó como larva de Anisakis sp. Posteriormente, en base a estudios morfométricos post diafanización en lactofenol de Aman, se identificó como Pseudoterranova decipiens (Figura 1).

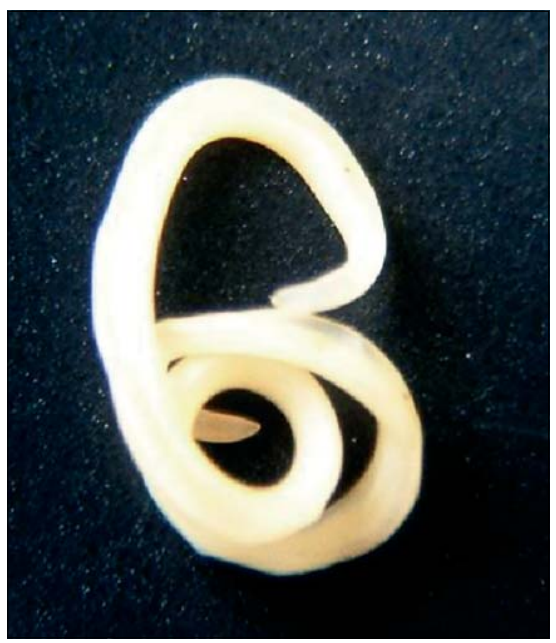

Figura 1. Larva eliminada por la paciente. 


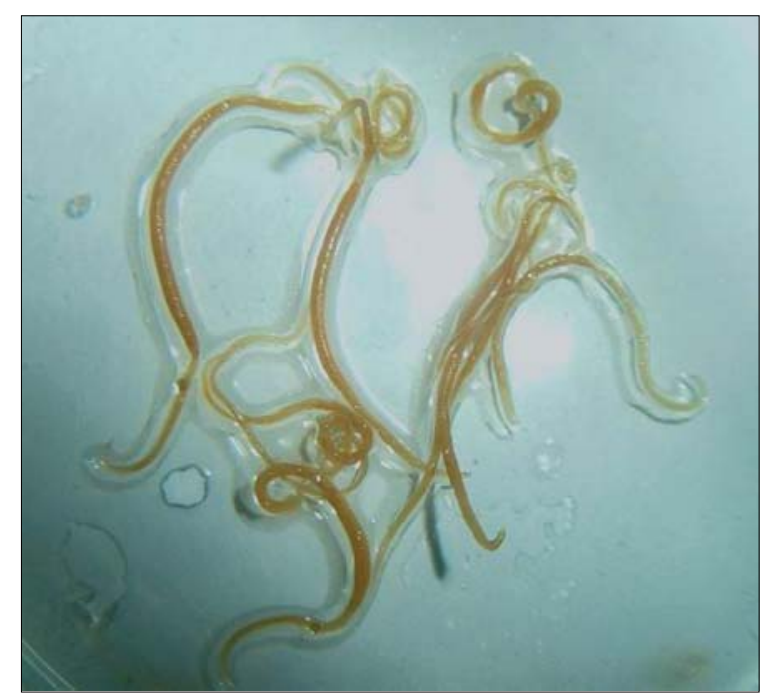

Figura 2. Larvas de Anisakis sp obtenidas de Seriola lalandii (vidriola).

\section{Discusión}

Los anisákidos pertenecen al Phylum Nemathelmintes, clase Nematoda, orden Ascaridida, familia Anisakidae. Comprende 24 géneros, los de mayor importancia son Anisakis, Contracaecum y Pseudoterranova.

La palabra anisakis deriva etimológicamente del griego anis, desigual y akis, punta. Son nemátodos, gusanos cilíndricos de extremos aguzados, de coloración blanquecina. Miden entre 2 y $3 \mathrm{cms}$, con un diámetro de 0,24 a $0,6 \mathrm{~mm}^{2}$ (Figuras 2 y 3).

La anisakidosis fue descrita por primera vez el año 1955 en los Países Bajos por Straub y publicada detalladamente en 1960 por van Thiel y cols, en relación al consumo de pescado ahumado. La anisakidosis es producida por larvas del tercer estadio de Anisakis simplex (anisakidosis o anisakiosis) o Pseudoterranova decipiens (pseudoterranovosis).

Estas infecciones parasitarias se adquieren por la ingesta de pescado crudo, ahumados al frío, salado, en vinagre, marinado o insuficientemente cocido, a la plancha o cocinados con microonda ${ }^{3}$. Se asocian principalmente al consumo de sushi, sashimi, cebiche y mariscal crudo.

Tanto A. simplex como P. decipiens se han encontrado en peces comercializados en el sur de Chile, con una intensidad media larva/músculo mayor para $P$. decipiens $^{5,6}$. En estudios realizados en 149 peces capturados en el litoral central de la Región de Valparaíso, se demostró la presencia de anisákidos en dos ejemplares de Sebastes capensis "cabrilla española" (33,3\%)

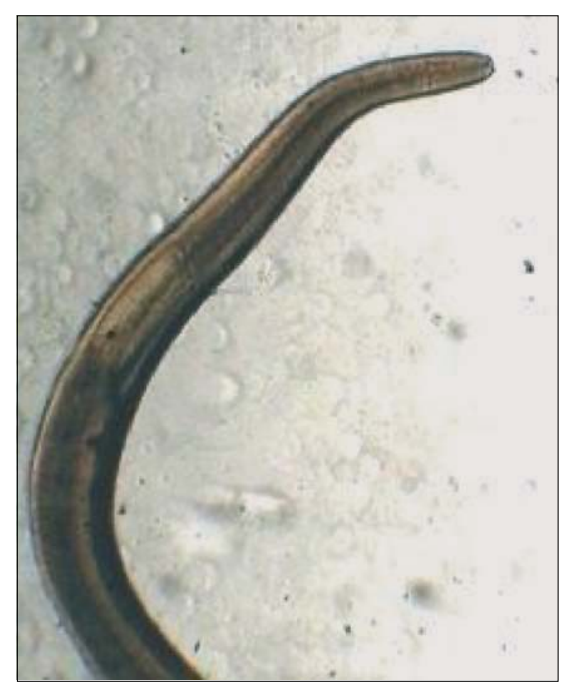

Figura 3. Extremo anterior de larva de Anisakis sp. y en un ejemplar de Paralabrax humeralis "cabrilla" (16,7\%) (Neira P., datos no publicados), en la isla Juan Fernández se encontró en 21,3\% de 80 ejemplares capturados, se detectó en 17 (41\%) Seriola lalandii "vidriola", usada para consumo interno y no comercializada (Grez M., datos no publicados).

En Japón es una parasitosis frecuente, por el alto consumo de pescado crudo, con más de 20.000 casos confirmados. En España es una infección endémica, al igual que en países americanos como Canadá, México y E.U.A. En Sudamérica se ha descrito esta parasitosis en Brasil, Chile y Perú. En Perú es considerada una enfermedad emergente, con ocho casos reportados, cifra probablemente sub-estimada por la falta de conocimientos del personal de salud de esta patología. Su presencia está asociada al fenómeno del niño y al aumento del consumo de pescado crudo ${ }^{7,8}$. En Brasil está demostrada la presencia de anisákidos en peces, con reportes de esta enfermedad ${ }^{9}$.

El primer caso de anisakidosis en nuestro país se describió el año $1976^{2}$, hasta ahora se han reportado 28 casos de anisakidosis, cuatro de ellos en pacientes pediátricos. La mayoría de los casos son producidos por $P$. decipiens ${ }^{4}$. En Bélgica se describió un caso de anisakidosis intestinal en un turista que adquirió la infección en un viaje a Chile y en Paraguay en un chileno que, por motivos de trabajo, viajó a ese país ${ }^{4,10}$. (Tabla 1).

Estos nematodos residen en el tracto digestivo de ballenas, delfines, focas, leones marinos y tiburones, entre otros. Los mamíferos excretan, junto con sus heces, huevos que se desarrollan en larvas de segun- 


\section{Tabla 1. Resumen de casos de anisakidosis y pseudoterranovosis reportados en Chile}

\begin{tabular}{|c|c|c|c|c|}
\hline Número de casos/ agente aislado & Año & Procedencia & Fuente & Evolución \\
\hline $\begin{array}{l}\text { N: } 15 / P \text {. decipiens. Incluye un paciente } \\
\text { de } 1 \text { año y otro de } 6 \text { años } \\
\text { (Ref. 4) }\end{array}$ & $2001-2005$ & $\begin{array}{l}\text { Santiago (11) } \\
\text { Cartagena (3) } \\
\text { Osorno (1) }\end{array}$ & $\begin{array}{l}\text { Cebiche (11) } \\
\text { Pescado frito (3) } \\
\text { Desconocido (1) }\end{array}$ & $\begin{array}{l}\text { Eliminación de la larva por la boca (13), } \\
\text { Eliminación por el ano (1) }\end{array}$ \\
\hline $\begin{array}{l}\text { N: } 1 / P \text {. decipiens } \\
\text { (Ref. 12) }\end{array}$ & 2006 & Limache & Cebiche & $\begin{array}{l}\text { Remoción de larva en esófago mediante } \\
\text { endoscopia }\end{array}$ \\
\hline $\begin{array}{l}\text { N: 1/ Anisakis sp en lactante de } 7 \text { meses } \\
\text { (Ref. 34) }\end{array}$ & 2003 & Santiago & $\begin{array}{l}\text { Ingesta de pescado } \\
\text { en sala cuna }\end{array}$ & $\begin{array}{l}\text { Cirugía laparoscópica: reducción de invagina- } \\
\text { ción y apendicectomía }\end{array}$ \\
\hline $\begin{array}{l}\text { N: } 1 / \text { Anisakis sp } \\
\text { (Ref. 35) }\end{array}$ & 2000 & San Antonio & Mariscal crudo & Eliminación por la boca \\
\hline $\begin{array}{l}\text { N: } 7 \text { / P. decipiens. } \\
\text { Incluye escolar de } 10 \text { años } \\
\text { (Ref 5) }\end{array}$ & $1997-1999$ & $\begin{array}{l}\text { Santiago (6) } \\
\text { Curicó (1) }\end{array}$ & $\begin{array}{l}\text { Cebiche (1) } \\
\text { Sushi (1) } \\
\text { Pescado frito (1) } \\
\text { Desconocido (4) }\end{array}$ & Eliminación por la boca (7) \\
\hline $\begin{array}{l}\text { N: } 1 / \text { P. decipiens } \\
\text { (Ref. 15) }\end{array}$ & 1995 & Coyhaique & Pescado ahumado & Remoción de la larva mediante endoscopia \\
\hline $\begin{array}{l}\text { N: 1/ Anisakis sp } \\
\text { (Ref. 13) }\end{array}$ & 1980 & Santiago & Consumo de jurel & Remoción de la larva mediante endoscopia \\
\hline $\begin{array}{l}\text { N: 1/ Phocanema sp } \\
\text { (Ref. 2) }\end{array}$ & 1976 & Santiago & Cebiche & $\begin{array}{l}\text { Eliminación de la larva por la boca (1), } \\
\text { eliminación por el ano post tratamiento (1) }\end{array}$ \\
\hline
\end{tabular}

do estadio, miden 0,3 mm de longitud y son infectantes. Las larvas pueden permanecer en el huevo o eclosionar y mantenerse en el agua, viables, por un período de tres meses. Son ingeridas por pequeños crustáceos (copépodos del plancton), donde se desarrollan alcanzando un tamaño de $5 \mathrm{~mm}$. Peces y cefalópodos ingieren estos crustáceos, convirtiéndose en los segundos hospederos intermediarios, las larvas migran y permanecen en las vísceras donde se desarrolla el tercer estadio. Pueden vivir en el pez por un año en anabiosis.

Se ha encontrado anisákidos en abadejo, arenque, bacalao, bonito, cabrilla, cojinova, congrio, jurel, lenguado, merluza, pescadilla, pejerrey, reineta, salmón, sardinas y vidriola. Así como en crustáceos como el

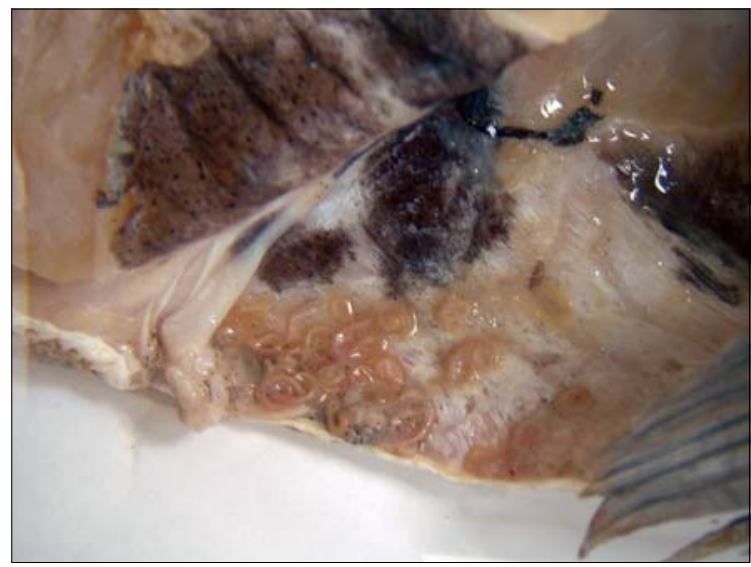

Figura 4. Larvas de Anisakis sp en vísceras. calamar nylon, langostino colorado y en el calamar. Por su forma y tamaño pueden pasar en forma inadvertida, si no se realiza una inspección visual detallada o se hace trans-iluminación de las vísceras (Figuras 4 y 5). Otra manera de detectar su presencia es por diferencia de conductividad del músculo y la larva.

El hombre es un hospedero accidental al ingerir peces crudos o mal cocidos que contienen la larva en estadio 3 y puede mudar a larva 4, pero no alcanza la madurez sexual ${ }^{11}$. Generalmente, es una larva única, que una vez ingerida ingresa al organismo y puede localizarse en el esófago ${ }^{12}$, estómago ${ }^{13}$, duodeno, yeyuno, ileon o colón ${ }^{14}$.

Existen ocho especies de Pseudoterranova: $P$.

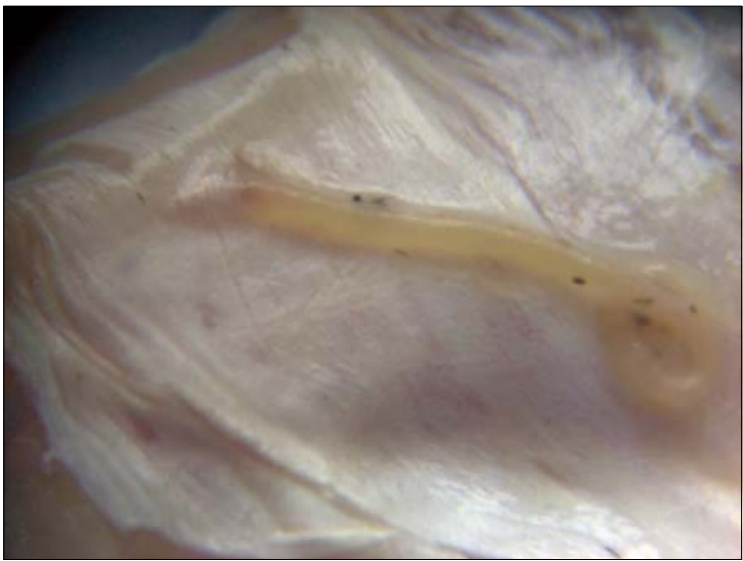

Figura 5. Larva de Anisakis sp en la musculatura. 
decipiens, P. karbie, P. bulbosa, P. azarasi, $P$. decipiens E, $P$. cattani, $P$. ceticola y $P$. kogiae. Los peces chilenos podrían portar más de una especie de Pseudoterranova ${ }^{4}$. Pseudoterranovosis decipiens se encuentra con mayor frecuencia en la musculatura más que en las vísceras. Se diferencia de Anisakis simplex desde el punto de vista morfológico ${ }^{15}$. La larva es de color amarillo rojizo, tiene una boca con tres labios, uno dorsal con dos papilas dobles y dos sub-ventrales con una papila doble. Sus medidas son: $2,8 \mathrm{~cm}$ de longitud, 0,8 cm de ancho. El esófago mide $1,8 \mathrm{~cm}$, el ventrículo $0,9 \mathrm{~cm}$, el ciego intestinal $0,8 \mathrm{~cm}$ y la cola 0,1 cm de longitud. Posee un diente cuticular cónico y prominente, dirigido hacia fuera. En el tubo digestivo, el ciego intestinal extendido hacia el extremo anterior sobrepasaba el margen anterior del ventrículo. En la cola se observa un mucrón cónico.

Anisakis simplex se encuentra con mayor frecuencia en climas tropicales y aguas cálidas, localizado en las vísceras de los hospederos, migrando rápidamente a la musculatura al momento de eviscerar el pescado. La larva del tercer estadio de $P$. decipiens se ha detectado en Chile en jurel, lenguado y merluza, y la forma adulta en mamíferos marinos. Los casos reportados de $P$. decipiens están asociados al consumo de cebiche, mariscos crudos, pescado frito y sushi ${ }^{4,6,12}$. La mayoría de los pacientes elimina el nemátodo en forma espontánea por la boca ${ }^{5}$. A diferencia de A. simplex, $P$. decipiens, rara vez migra al estómago, es menos invasor de la pared gastrointestinal y ocasiona pocos síntomas ${ }^{16-18}$.

Anisakis simplex puede producir una reacción alérgica grave, que se presenta entre cinco y 26 horas después de la ingesta, usualmente a las cinco horas ${ }^{19}$. Las formas de presentación de esta parasitosis son gástrica (95,6\%), intestinal (4\%) y extra-intestinal (0,4\%). La presentación clínica más frecuente es una gastritis aguda, caracterizada por un dolor epigástrico intenso y repentino, asociado a náuseas y vómitos. El dolor se inicia entre una y 12 horas después de la ingesta, con rangos de hasta 14 días. Si se realiza una endoscopia precozmente se puede visualizar la larva en la mucosa gástrica, generalmente viva y parcialmente introducida; si es realizada posteriormente, se va a encontrar erosión de la mucosa, placas inflamatorias con presencia de eosinófilos y granulomas; en estos casos se recupera la larva en forma parcial ${ }^{20-22}$. A veces se producen accesos de tos con eliminación del ejemplar, sin otros síntomas acompañantes. Ocasionalmente puede eliminarse por deposiciones. La larva muere en el transcurso de siete meses.

La respuesta alérgica de $A$. simplex es mediada por antígenos excretorios y secretorios de la larva viva; no obstante, se ha visto en pacientes que han ingerido pescado parasitado, debidamente cocido. En Japón, uno de los países de mayor prevalencia de anisakidosis, en $63 \%$ de la población puede encontrarse intradermoreacción positiva a $A$. simplex; en España, en 15,2\% de los donantes de sangre ${ }^{23}$.

En algunos pacientes se produce la llamada anisakidosis gastro-alérgica, que es una reacción aguda mediada por IgE, inducida por la larva viva que penetra a la mucosa gástrica. Los síntomas alérgicos se acompañan de dolor epigástrico que puede ser de intensidad leve o estar ausente; en ellos se rescata el antecedente de ingesta, horas antes, de pescado crudo. Puede producirse urticaria y el angioedema en $10 \%$ de los casos de anisakidosis, e incluso, reacción anafiláctica con shock ${ }^{24}$. En pacientes con urticaria crónica, en áreas endémicas de esta parasitosis, se encuentra intradermo-reacción positiva.

Otras formas de presentación de anisakidosis son la obstrucción e invaginación intestinal ${ }^{25}$, estenosis intestinal ${ }^{26}$, peritonitis ${ }^{27}$, asma ocupacional ${ }^{28}$ y manifestaciones reumatológicas como artralgia y artritis ${ }^{29}$. La larva móvil puede penetrar la pared intestinal, invadir y llegar al hígado, bazo, páncreas ${ }^{30}$, pulmones ${ }^{31}$ y ovario. Se ha asociado a conjuntivitis, faringitis recurrente y adenopatías mesentéricas ${ }^{23,32-33}$.

Tanto anisakidosis como pseudoterranovosis se presentan predominantemente en adultos, con escasos reportes en Pediatría ${ }^{34}$. En la mitad de los casos se puede encontrar eosinofilia en el hemograma. La mayoría de los pacientes se recupera espontáneamente, en otros debe realizarse una endoscopia digestiva alta para la remoción de la larva ${ }^{35}$. La cirugía está indicada en casos de obstrucción intestinal y peritonitis por perforación. Hay experiencias anecdóticas con el tratamiento con albendazol, en dosis diaria de 400 a 800 mg durante 6 a 21 días $^{36-38}$. El consumo de pescado crudo por perros y gatos, puede favorecer el desarrollo de anisákidos en estos animales ${ }^{6}$.

El sushi y el sashimi son preparados tradicionales de la comida japonesa en base a pescados crudos, por lo que representan un importante riesgo ${ }^{39}$. La principal medida de prevención de esta parasitosis es no consumir pescados crudos o insuficientemente cocidos. Se deben eviscerar en forma rápida en alta mar, para evitar la migración de las larvas a la musculatura y eliminar los peces parasitados. La larva puede inactivarse con cocción a temperaturas sobre $60^{\circ} \mathrm{C}$ durante al menos 15 minutos, congelación a $-20^{\circ} \mathrm{C}$ por más de una semana, congelación rápida a $-20^{\circ} \mathrm{C}$ durante 48 a 72 horas o a $-35^{\circ} \mathrm{C}$ por 15 horas. La cocción en horno microonda requiere de una temperatura mayor a $77^{\circ} \mathrm{C}$ en la porción más gruesa del producto ${ }^{4,13,20}$.

En China, estos productos se consumen con jengibre que inactivarían las larvas cuando son expuestos 
durante más de 17 horas. En salsa de soya pueden resistir la inmersión por 18 horas. El wasabi (Eutrema wasabi) en solución al 5\% o pasta comercial puede destruir la larva en dos horas. En soluciones salinas al $1-2 \%$ sobrevive por más de seis meses, al 4,3\% siete semanas y al 8-9\% cinco a seis semanas. En ácido acético al 1\% más de tres meses ${ }^{17,39-42}$.

Si bien existe una normativa nacional, artículo 323 del Reglamento Sanitario de alimentos del año 1997, que se refiere a la desparasitación de los pescados usados para consumo, no contamos con una norma actualizada para la notificación de las enfermedades parasitarias transmitidas por alimentos.

\section{Resumen}

Se comunica un caso de ingestión accidental de Pseudoterranova decipiens. A raíz de esta paciente se revisan los principales conceptos relativos a esta naciente patología en Chile. El consumo de platos prepa- rados con pescados crudos como el sushi, sashimi, cebiche y mariscal es cada vez más popular. A través de la ingesta de hospederos intermediarios usados en la preparación, se puede adquirir la larva de tercer estadio de Anisakis simplex o $P$. decipiens. Estos parásitos pueden provocar gastritis aguda que se manifiesta inmediatamente después de consumido el pescado crudo. En ocasiones puede haber compromiso intestinal e invasión del tracto gastrointestinal con manifestaciones en otros órganos. Se asocia a manifestaciones alérgicas como urticaria, angioedema y shock anafiláctico. La larva se elimina en forma espontánea en la mayoría de los casos, pero en otros debe removerse por vía endoscópica. En Chile, es más frecuente la presencia de $P$. decipiens, que por lo general no migra a la mucosa gástrica, no invade y produce pocos síntomas. Las medidas de prevención son no comer pescados crudos, cocinarlos a temperaturas elevadas o congelación previa a la preparación de los alimentos, para inactivar las larvas.

\section{Referencias}

1.- Butt A A, Aldridge K E, Sanders C V. Infections related to the ingestion of seafood. Part II: parasitic infections and food safety. Lancet Infect Dis 2004; 4: 294-300.

2.- Sapunar J, Doerr E, Letonja F. Anisakiasis humana en Chile. Bol Chil Parasitol 1976; 31: 79- 83

3.- Cnut Blasco A, Labora Lóriz A, López de Torre J, Romeo Martínez J A. Anisakiasis gástrica aguda por cocción insuficiente en horno microondas. Med Clin 1996; 106: 317-18.

4.- Torres P, Jercic M I, Weitz J C, Dobrew E K, Mercado R. Human pseudoterranovosis, an emerging infection in Chile. J Parasitol 2007; 93: 440-3.

5.- Mercado R, Torres P, Muñoz V, Apt W. Human infections by Pseudoterranova decipiens (Nematoda, anisakidae) in Chile: report of seven cases. Mem Inst Oswaldo Cruz, Río de Janeiro 2001; 96: 653-55.

6.- Torres P, Moya R, Lanilla J. Nematodos anisákidos de interés en salud pública en peces comercializados en Valdivia, Chile. Arch Med Vet 2000; 32: 107-13.

7.- Cabrera R, Luna-Pineda MA, Suárez-Ognio L. Nuevo caso de infección humana por una larva de Pseudoterranova decipiens (Nematoda, Anisakidae) en el Perú. Rev Gastroenterol Perú 2003, 23: 217-20.

8.- Cabrera R, Trillo-Altamirano M P.
Anisakidosis: ¿ ¿na zoonosis parasitaria marina desconocida o emergente en el Perú? Rev Gastroenterol Perú. 2004; 24: 335-42.

9.- Amato Neto V, Amato J G P, Amato V S. Probable recognition of human anisakiasis in Brazil. Rev Inst Med Trop S Paulo 2007; 49: 261-2.

10.- Verhamme M A, Ramboer C H R. Anisakiasis caused by herring in vinager: a little know problem. Gut 1988; 29: 843-7.

11.- Rosales M J, Mascaró C, Fernández C, Luque F, Sánchez Moreno M, Parras L, et al. Acute intestinal anisakiasis in Spain: a fourth-stage Anisakis simplex larva. Mem Inst Oswaldo Cruz, Río de Janeiro 1999; 94: 823-6.

12.- Mercado R, Torres P, Gil L C, Goldin L. Anisakiasis en una paciente portadora de una pequeña hernia hiatal. Rev Méd Chile 2006; 134: 1562- 4.

13.- Apt W, Hisamoto T, Llorens P, Alcaíno H. Anisakiasis gástrica en Chile. Rev Méd Chile 1980; 108: 825- 7.

14.- Gill C J, Harner D H. An uninvited dinner guest. Clin Infect Dis 2005; 41: 1810-1.

15.- Mercado R, Torres P, Maira J. Human case of gastric infection by a fourth larval stage of Pseudoterranova decipiens (Nematode, Anisakidae). Rev Saúde Publica 1997; 31: 178-81.

16.- Chinda D, Menamoto K, Shimoyama T, Sasaki H, Nara H, Munakata A. Asymptomatic colonic anisakiosis identified during colonoscopy. Digestive
Endosc 2004; 16: 176-7.

17.- Sakanari J A, Mckerrow J H. Anisakiasis. Clin Microbiol Rev 1989; 2: 287-84.

18.- Weir E. Sushi, nematodes and allergies. Can Med Assoc J 2005; 172: 329.

19.- Takei H, Powell S Z. Intestinal anisakidosis (anisakiosis). Ann Diag Pathol 2007; 11: 350-2.

20.- Shiomi M, Kamisako T, Yutani I, Yoshimoto R, Kudo M, Fujii R. Anisakis in a biopsy specimen from the edge of a gastric ulcer: report of a case. Gastrointestinal Endosc 2004; 60: 854-5.

21.- López- Serrano M C, Alonso-Gómez A, Daschner A, Moreno-Ancillo A, Suárez de Parga J M, Caballero M T et al. Gastroallergic anisakiasis. Findings in 22 patients. J Gastroenterol Hepatol 2000; 15: 503-6.

22.- Hokama A, Gakiya I, Miyagi T, Fukuchi J, Kinjo F, Saito A. Gastrointestinal acute gastric anisakiasis. J Gastroenterol Hepatol 2005; 20: 1121.

23.- Daschner A, Pascual C Y. Anisakis simplex: sensitization and clinical allergy. Curr Opin Allergy Clin Immunol 2005; 5: 281-5.

24.- Daschner D, Alonso-Gómez A, Caballero T, Barranco P, Suárez de Pargua J M, LópezSerrano M C. Gastric anisakiasis: an underestimated cause of acute urticaria and angio-oedema. Br J Dermatol 1998; 139: 822-8.

25.- García-Labairu C, Alonso-Martínez J L, Martínez Echeverría A, Rubio-Vela T, 
Zozaya-Urmeneta J M. Asymptomatic gastroduodenal anisakiasis as the cause of anaphylaxis. Eur J Gastroenterol Hepatol 1999; 11: 785-7.

26. - Ishida M, Harade A, Egawa S, Watabe S, Ebina N, Unno M. Three succesives cases of enteric anisakiasis. Dig Surg 2007; 24: 228-31.

27.- Matsuo S, Azuma T, Susumu S, Yamaguchi S, Obata S, Hayashi T. Small bowel anisakiosis: a report of two cases. World J Gastroenterol 2006; 12: 4106-8.

28. - Onta M, Ikeda K, Miyakoshi H, Nishide K, Horigami T, Akao T, et al. A very rare cause of continuous ambulatory peritoneal dyalisis peritonitis caused by Anisakis larva. Am J Gastroenterol 1995; 90: 1902-3.

29.- Nieuwenhuizen N, Lopata A L, Jeebhay M F, Herbert D R, Robin T G, Brombacher F. Exposure to the fish parasite Anisakis cause allergic airway hiperreactivity and dermatitis. J Allerg Clin Immunol 2006; 117: 1098105.

30.- Audicana M T, Ansotegui I J, Fernández de Corres L, Kennedy M W. Anisakis simplex: dangerous- dead and alive? Trends Parasitol 2002; 18: 20-5.

31.- Pezzilli R, Casadei R, Santini D. Autoimmune pancreatitis associated with anisakis infection. Dig Liver Dis 2007; 39: 273.

32.- Saito W, Kawakami K, Matsuo H, Oishi K, Nagatake T. Pulmonary anisakiasis presenting as eosinophilic pleural efussion. Respirology 2005; 10: 261-2.

33.- Añíbarro B, Seoane F J. Occupational conjuctivitis caused by sensitization to Anisakis simplex. J Allergy Clin Immunol 1998; 102: 331-2.

34.- Castillo C, Martínez V, Ossandón F. Anisakiasis en un lactante. Rev Chil Pediatr 2003; 74: 415-6.

35.- Torres M, Canales M, Concha M, Cofré X, Tellez P. Un caso de anisakiosis en un adulto. Parasitol Día 2000; 24: 109-11.

36.- Pacios E, Arias-Díaz J, Zuloaga J, GonzálezArmengol J, Villarroel P, Balbrea J L. Albendazole for the treatment of anisakiasis ileus. Clin Infect Dis 2005; 4: 1825-6.

37.- Moore D, Girdwood R, Chiodini P.
Treatment of anisakiasis with albendazole. Lancet 2002; 360:54.

38. - Arias-Díaz J, Zuloga J, Vara E, Balibrea J, Balibrea J L. Efficacy of albendazole against Anisakis simplex larvae in vitro. Dig Liver Dis 2006; 38: 24-36.

39. - Nawa Y, Hatz C, Blurn J. Sushi delights and parasites: the risk of fishborne and foodborne parasitic zoonoses in Asia. Clin Infect Dis 2005; 41: 1297-303.

40.- Smith J W, Wootten R. Anisakis and anisakiasis. Adv Parasitol 1978; 16: 93-163.

41.- Murata I, Miyazawa S, Kuni M, Nakajima Y, Sibuya T, Nakarishi H. Inhibitory effects of partial freezing, several kinds of condiments, species and seasonings on the activity of Anisakis type I larvae collected from Teragra chalcogramma and Pneumatophorus japonicus. Annual Report of the Tokyo Metropolitan Research Laboratory of Public Health 1987; 38: 13-21.

42.- Beldsoe G E, Oria M P. Potential hazards in cold smoked fish: parasite. J Food Science 2001; 66 (Suppl 7): S1100-3.

\title{
Comentario Editorial Pseudoterranovosis
}

\author{
Patricia Muñoz C. del V.
}

$\mathrm{U}$

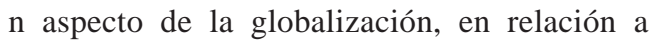
hábitos culinarios, ha sido el aumento del consumo de pescados y mariscos crudos, muy propios de la cocina asiática, especialmente de la japonesa, que ha traído como consecuencia una enfermedad parasitaria emergente como es la anisakidosis. Esta parasitosis accidental del hombre es producida por nemátodos anisákidos (familia Anisakidae) cuyos huéspedes definitivos habituales son mamíferos marinos ictiófagos y en cuyo ciclo intervienen crustáceos y peces, muchos de ellos de importancia comercial, que forman parte de la cadena alimenticia y en cuyo interior se desarrollan los estados larvales de estos parásitos.

El hombre, al consumir pescados crudos o ahumados en frío, puede desarrollar una pseudoterranovosis (Pseudoterranova decipiens u otras especies) o una anisakiosis (Anisakis simplex u otras especies) según el género involucrado. En el primer caso las larvas, al parecer, no penetran en los tejidos, siendo muchos de los afectados asintomáticos, eliminan las larvas con la tos o bien, presentan síntomas en forma muy precoz que se manifiestan por dolor epigástrico, náuseas y vómitos. En el segundo caso, las larvas pueden penetrar en distintos segmentos del tracto gastrointestinal ocasionando molestias de diversa índole y magnitud según el segmento comprometido y el grado de hipersensibilidad desarrollado.

El diagnostico del género y especie involucrada se puede establecer por estudios de la región cefálica, de las papilas caudales y de diversas variables morfométricas de estos vermes. El laboratorio de Parasitología del Instituto de Salud Pública de Chile, como así también los laboratorios de Parasitología de diversas universidades chilenas, se encuentran capacitados para realizar este diagnóstico diferencial.

Si bien a nivel mundial, es más frecuente la anisakiosis, en un estudio realizado por Torres P. y colaboradores en peces comercializados en Valdivia, se identificaron más especies de peces y con mayor intensi-
Editora sección Parasitología Hospital Militar del General Luis Felipe Brieba Arán

Correspondencia a: Patricia Muñoz C. del V. 